\title{
Satisfação com a vida e satisfação diádica: correlações entre construtos de bem-estar
}

\author{
Fabio Scorsolini-Comin ${ }^{1}$ - Universidade de São Paulo, Ribeirão Preto, Brasil \\ Manoel Antonio dos Santos² - Universidade de São Paulo, Ribeirão Preto, Brasil
}

\begin{abstract}
Resumo
A literatura científica aponta que pessoas casadas tenderiam a ser mais felizes do que as não casadas. Este estudo teve por objetivo investigar as relações existentes entre satisfação com a vida e satisfação diádica em pessoas casadas. Participaram 106 pessoas casadas, de ambos os sexos, com média de idade de $42 \pm 11$ anos. Foram utilizados instrumentos para caracterização sociodemográfica e classificação socioeconômica, Escala de Bem-Estar Subjetivo e Escala de Ajustamento Diádico. Pela análise de correlações e de regressão múltipla stepwise, verificou-se que a satisfação com a vida (variável do construto BES) e a satisfação diádica (variável de conjugalidade) são positiva e significativamente correlacionadas $(r=0,20 ; \mathrm{p}=0,04)$, o que revela que pessoas que se dizem satisfeitas com a vida, em diferentes domínios também o fazem em relação à experiência conjugal.

Palavras-chave: Felicidade, Satisfação, Relações conjugais, Casamento, Escala de Bem-Estar Subjetivo.
\end{abstract}

\section{Life satisfaction and dyadic satisfaction: correlations between constructs of well-being}

\begin{abstract}
Scientific literature points out that married people tend to be happier than non-married people. The objective of this present study was to investigate the relationship between life satisfaction and dyadic satisfaction among married people. 106 married people (53 couples), with an average age of $42(\mathrm{sd}=11)$, participated in the study. The following instruments were used: demographic identification and social classification; Subjective Well-being Scale; and Dyadic Adjustment Scale. Correlation analysis and stepwise multiple regression analysis showed that satisfaction with life and dyadic satisfaction are positive and significantly correlated $(\mathrm{r}=0.20 ; \mathrm{p}=0.04)$. Based on this finding, the researchers have concluded that people who are generally satisfied with life are also satisfied in the conjugal relationship.

Keywords: Happiness, Satisfaction, Marital relations, Marriage, Subjective Well-Being Scale.
\end{abstract}

A literatura científica destaca que pessoas casadas de ambos os sexos relatam ter mais felicidade do que aquelas que nunca casaram ou são divorciadas, separadas ou viúvas. Pessoas que coabitam com um parceiro também são significativamente mais felizes em algumas culturas do que aquelas que vivem sozinhas. As pessoas mais felizes também são mais propensas a se casar e construir um relacionamento afetivo mais satisfatório (Albuquerque, 2004; Argyle, 1999; Diener \& Lucas, 2000; Galinha, 2008; Lee, Seccombe \& Shehan, 1991; Scorsolini-Comin, 2009; Snyder \& Lopez, 2009).

Seligman (2004), considerado o fundador da Psicologia Positiva na década de 1990, atesta que o casamento está diretamente relacionado à felicidade. Retomando os resultados de uma pesquisa realizada nos últimos 30 anos, com 35 mil pessoas nos Estados Unidos, $40 \%$ das pessoas casadas "se diziam muito felizes, enquanto apenas $24 \%$ das divorciadas, solteiras, separadas e viúvas tinham essa opinião" (Seligman, 2004, p. 72). Ainda segundo o autor, viver com a pessoa amada (sem que haja o casamento consensual) também é um fator que está diretamente relacionado à felicidade. Comparativamente, pessoas que não vivenciaram tal experiência apresentam menores taxas

\footnotetext{
1 E-mail: scorsolini_usp@yahoo.com.br

Endereço para correspondência:

${ }^{2}$ Departamento de Psicologia e Educação - Faculdade de Filosofia, Ciências e Letras de Ribeirão Preto - Universidade de São Paulo USP

Avenida Bandeirantes, 3.900 - Monte Alegre - 14040-901 Ribeirão Preto-SP
}

de felicidade. Esses dados também são corroborados por Snyder e Lopez (2009), que retomaram as clássicas investigações realizadas no âmbito da psicologia positiva e que buscavam, em sua essência, comprovar que as pessoas com maior nível de felicidade tenderiam a estabelecer relacionamentos afetivos mais seguros, com maior troca de experiências positivas e, consequentemente, mais satisfatórios.

Seligman (2004) levanta as hipóteses de que pessoas felizes têm mais possibilidades de se casarem e manterem o casamento ou de que existiria uma terceira variável (como boa aparência e sociabilidade), que seria a causa de maior felicidade e probabilidade de um casamento. Desse modo, para esse autor o casamento está associado à felicidade, mas ainda não são claras as variáveis presentes no casamento (ou nos cônjuges) que estariam associadas a essa avaliação de bem-estar.

Segundo Perlin (2006), casamento e satisfação ficaram, ao longo da história do ocidente, estreitamente interdependentes. O casamento, dentro de nossa estrutura política e econômica, tem sido definido como uma resultante social que satisfaz necessidades básicas do indivíduo. Dias (2000) afirma que o casamento contemporâneo tem algumas características determinantes, entre as quais está a busca da felicidade, da satisfação e do amor. O desejo intenso de estar com o outro motiva o casamento e determina a escolha do parceiro, pois "os indivíduos esperam encontrar nesses relacionamentos uma compatibilidade afetiva, sexual e intelectual" (Perlin, 2006, p. 66).

Como destacado por Perlin (2006), a satisfação é um elemento fundamental em um 
relacionamento interpessoal. A partir dessa premissa, poderíamos supor que quão mais satisfeitas as pessoas se sentem no casamento, mais seriam felizes também em termos individuais, ou seja, apresentariam maior nível de bem-estar subjetivo (BES), notadamente em termos do construto satisfação com a vida.

Uma das escalas mais utilizadas para mensurar a satisfação conjugal é a Dyadic Adjustment Scale (DAS). Essa escala foi desenvolvida por Spanier (1976) com base no conceito de ajustamento conjugal, tendo sido utilizada em uma série de estudos nacionais e internacionais não apenas para avaliar o ajustamento conjugal (Ramos \& Patrão, 2005; Scorsolini-Comin, 2009), mas também para que fossem apuradas as suas características psicométricas (Carey, Spector, Lantinga \& Krauss, 1993; Fisiloglu \& Demir, 2000; Graham, Liu \& Jeziorski, 2006; Hernandez, 2008). No Brasil, o estudo de Hernandez (2008) mostrou que a versão da escala para a língua portuguesa (Escala de Ajustamento Diádico) forneceu uma medida confiável e válida para avaliar ajustamento diádico no contexto nacional em atividades de investigação psicológica.

De acordo com Spanier (1976), o ajustamento conjugal pode ser visto em duas perspectivas distintas: como um processo ou avaliação qualitativa de um estado. Para este autor, definir ajustamento conjugal como um processo tem diversas implicações, sendo a mais importante delas a de que um processo pode ser mais bem estudado ao longo do tempo. Segundo Hernandez e Hutz (2008), os estudos transversais na investigação do ajustamento têm algum valor, contudo, é evidente que um processo pode ser mais bem observado mediante delineamentos longitudinais.

As dimensões do ajustamento conjugal são: consenso diádico, coesão diádica, expressão do afeto e satisfação diádica. A satisfação diádica, especificamente investigada no presente estudo, mede a percepção das questões relativas à discussão do divórcio, à saída de casa após briga, ao arrependimento com o casamento, à implicância mútua, ao estar bem, à confiança no cônjuge, ao beijo no cônjuge, ao grau de felicidade e ao compromisso com o futuro do relacionamento (Hernandez, 2008; Hernandez \& Hutz, 2008; Magagnin, Körbes, Hernandez, Cafruni, Rodrigues \& Zarpelon, 2003).

No presente estudo, em consonância com a abordagem da psicologia positiva, a felicidade é concebida em sua matriz científica, sob o conceito de bem-estar subjetivo (BES). O BES, ou o estudo científico da felicidade (Seligman, 2004), é uma noção que visa compreender a avaliação que as pessoas fazem de suas vidas (Costa \& Pereira, 2007). Apesar das discordâncias teóricas relativas à conceituação de BES, há um consenso quanto às suas dimensões: satisfação com a vida e afetos positivos e negativos (Anguas, 1997; Diener, Suh \& Oishi, 1998; Glatzer, 1987; Martinez \& Garcia, 1994; Resende, Bones, Souza e
Guimarães, 2005). Os afetos positivos seriam as sensações transitórias de êxtase, interesse, alegria, contentamento, prazer, ao passo que os afetos negativos envolveriam experiências emocionais de desprazer, tristeza, depressão, decepção, frustração, entre outras.

A dimensão satisfação com a vida corresponde a um julgamento cognitivo de algum domínio específico na vida da pessoa; um processo de juízo e avaliação geral da própria vida (Emmons, 1986); uma avaliação sobre a vida de acordo com um critério próprio (Shin \& Johnson, 1978). Esse julgamento depende de uma comparação entre as circunstâncias de vida do indivíduo e um padrão por ele escolhido, ou seja, remete também a uma comparação com o seu meio social, cultural e histórico.

Por outro lado, o construto satisfação diádica examina as percepções individuais acerca da possibilidade do divórcio/separação, da evasão de casa, do arrependimento, das querelas, da implicância mútua, do bem-estar, da confiança, do beijo, da felicidade e do compromisso com o relacionamento (Hernandez, 2008; Hernandez \& Hutz, 2008). Uma questão que ainda requer maior esclarecimento é como os construtos de satisfação com a vida e satisfação diádica podem ser correlacionados.

Tendo em vista esses questionamentos, o presente estudo teve por objetivo investigar as relações existentes entre satisfação com a vida e satisfação diádica em pessoas casadas. Como a literatura aponta que pessoas casadas tendem a ser mais felizes, a hipótese principal do estudo é de que existem correlações positivas e significativas entre satisfação com a vida e satisfação diádica.

\section{Método}

\section{Participantes}

Este estudo de caráter descritivo, transversal, do tipo correlacional, contou com a participação de voluntários casados, oriundos de região urbana, de um município do interior do estado de São Paulo. A amostra foi composta por 106 participantes legalmente casados há, no mínimo, um ano.

Tais participantes foram encontrados pela técnica da "bola de neve", em que novos participantes foram indicados pelos próprios respondentes, a partir dos contatos do pesquisador. Todos foram, inicialmente, esclarecidos sobre os objetivos do trabalho por meio da leitura do Termo de Esclarecimento ao Participante. Após concordarem em participar, formalizaram sua anuência por meio de assinatura do Termo de Consentimento Livre e Esclarecido, em conformidade com os aspectos éticos preconizados para pesquisas com seres humanos. $\mathrm{O}$ projeto foi aprovado por Comitê de Ética em Pesquisa (protocolo $\mathrm{n}^{\circ}$ 2007.1.2016.59.0). 


\section{Instrumentos}

Quanto aos instrumentos para a coleta de dados, foi utilizada uma bateria de escalas e questionários, descritos a seguir. Para identificação geral do participante foram utilizados: (a) Questionário Sociodemográfico para caracterização dos participantes; (b) Classificação Socioeconômica. O critério Abipeme (1997) é uma escala ou classificação socioeconômica construída por intermédio da atribuição de pesos a um conjunto de itens de conforto doméstico, além do nível de escolaridade do chefe de família.

Para avaliação da satisfação com a vida utilizou-se a Escala de Bem-Estar Subjetivo - EBES: instrumento inspirado em escalas preexistentes, propostas originalmente na língua inglesa: Escala de Afeto Positivo e Afeto Negativo - PANAS, Escala de Satisfação com a Vida - SWLS e Escala de Bem-Estar Subjetivo - SWBS. É composta por três fatores: afeto positivo (21 itens e índice de consistência interna alfa de Cronbach de 0,95), afeto negativo (26 itens e alfa de Cronbach de 0,95$)$ e satisfação com a vida (15 itens e alfa de Cronbach de 0,90). O instrumento é composto por duas subescalas de resposta tipo Likert de cinco pontos. $\mathrm{Na}$ primeira parte da escala, os itens vão do número 1 ao 47 e descrevem afetos positivos e negativos, devendo o sujeito responder como tem se sentido ultimamente em uma escala em que 1 significa nem um pouco e 5 significa extremamente. $\mathrm{Na}$ segunda parte da escala, os itens vão do número 48 ao 62 e descrevem julgamentos relativos à avaliação de satisfação ou insatisfação com a vida, devendo ser respondidos em uma escala na qual 1 significa discordo plenamente e 5 significa concordo plenamente. Esse instrumento foi validado para o contexto brasileiro por Albuquerque e Tróccoli (2004).

Para avaliação da satisfação diádica utilizou-se a Escala de Ajustamento Diádico - DAS: escala desenvolvida por Spanier (1976) para avaliação da percepção de casais acerca de seus relacionamentos afetivos. É utilizada internacionalmente, com adaptações para vários países e culturas (Fisiloglu \& Demir, 2000; Hernandez, 2008), composta por 32 itens. Possui quatro domínios, a saber: consenso (13 itens), coesão (cinco itens), satisfação (10 itens) e expressão do afeto (quatro itens). No estudo original (Spanier, 1976), as propriedades psicométricas encontradas, no que se refere à consistência interna da escala, foram: alfas de Cronbach de 0,90 (consenso diádico), 0,94 (satisfação diádica), 0,86 (coesão diádica) e 0,73 (expressão do afeto) (Spanier, 1976). Em estudo brasileiro, o alfa de Cronbach total da escala foi de 0,93 (Hernandez, 2008).

\section{Procedimento de análise de dados}

Para a análise dos resultados, os dados obtidos por meio da aplicação dos instrumentos foram transpostos para o software $\mathrm{R}$ ( $\mathrm{R}$ Development Core Team, 2005) e categorizados. Como o objetivo foi comparar se houve evidências de diferença na distribuição dos domínios segundo algumas variáveis explicativas, utilizou-se o teste não-paramétrico para amostras independentes de Kruskal-Wallis ou, no caso de dois níveis para a variável explicativa, o teste de Wilcoxon, também para amostras independentes.

A força da grandeza do coeficiente de correlação foi avaliada conforme procedimento proposto por Zou, Tuncali e Silverman (2003). O nível de significância adotado foi de $p \leq 0,01$. Depois do estabelecimento das correlações, buscou-se estabelecer a relação dos escores totais dos diversos domínios segundo um modelo linear múltiplo. Foi utilizado o método de seleção de variáveis stepwise, em que permaneceram no modelo aquelas que apresentaram maior evidência de significância do ponto de vista multivariado (Montgomery, Vining \& Peck, 2001).

\section{Resultados e Discussão}

Quanto à caracterização dos participantes, a

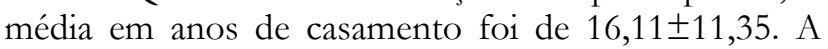
média de idade foi de $42 \pm 11$ anos. A média de idade dos homens foi de 43,4 anos e a das mulheres, 40,7. Em relação ao número de filhos dos casais

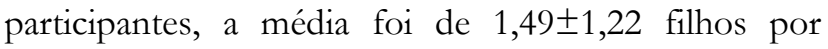
participante. Em termos da classificação socioeconômica, aferida a partir do critério Abipeme, a maioria pertencia à classe B $(60,37 \%)$, com renda per

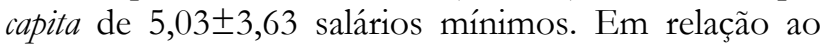
grau de instrução dos participantes, a maioria $(66,98 \%)$ possuía nível superior, o que se observou tanto entre homens quanto entre mulheres.

A satisfação diádica não apresentou associações com a idade dos participantes ( $\mathrm{r}=-0,15$; $\mathrm{p}=0,12)$, tempo de relacionamento dos casais $(\mathrm{r}=-0,17$; $\mathrm{p}=0,21)$, grau de instrução $(\mathrm{p}=0,13)$, classificação socioeconômica $(\mathrm{p}=0,11)$ e renda per capita $(\mathrm{r}=0,08$; $\mathrm{p}=0,59)$.

Analisando-se a satisfação com a vida (variável do construto $\mathrm{BES}$ ) e a satisfação diádica (variável de conjugalidade), observou-se que as mesmas estão positiva e significativamente correlacionadas $(\mathrm{r}=0,20$; $\mathrm{p}=0,04)$, o que revela que pessoas que se dizem satisfeitas com a vida em diferentes domínios também o fazem em relação à experiência conjugal (Figura 1). 


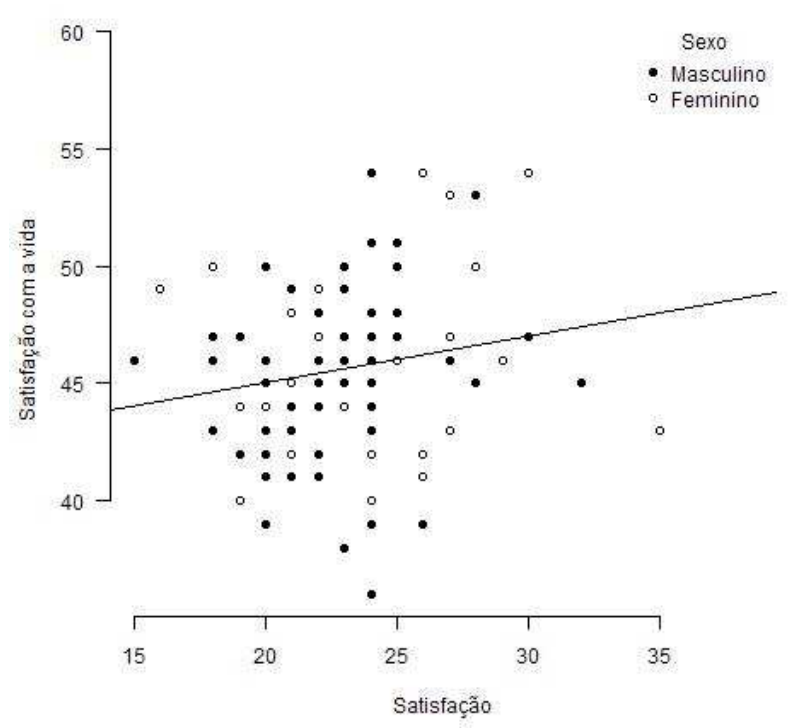

Figura 1 - Correlação entre a satisfação com a vida e a satisfação diádica $(N=106)$.

Tal consideração confirma a hipótese deste estudo, na medida em que ambos os domínios, por avaliarem o julgamento da pessoa sobre o que vivencia (em sua vida de casada ou não) em relação aos seus desejos e expectativas construídas cultural e socialmente, manteriam uma relação de semelhança na definição dos construtos. A satisfação com a vida, por envolver um julgamento global, também abarca os relacionamentos afetivos. Assim, a satisfação conjugal, na amostra, está positiva e significativamente correlacionada com a satisfação com a própria vida. Este apontamento é corroborado também pela análise de regressão múltipla tipo stepwise.
Ainda por essa análise, a satisfação com a vida também seria influenciada diretamente pelos afetos positivos e negativos, corroborando a consistência interna do construto. Ou seja, para que uma pessoa faça uma avaliação de sua vida e um julgamento pessoal em termos de satisfação com as diversas dimensões que o compõem, precisa ter bom nível de afetos positivos e baixo nível de afetos negativos, como destacado na Tabela 1. Por outro lado, a satisfação com a vida mostra-se correlacionada com a variável idade.

Tabela 1 - Matriz de correlação e p-values independentemente da variável sexo. Os valores destacados em negrito foram os considerados significativos

\begin{tabular}{lcccc}
\hline & Afetos positivos & Afetos negativos & Satisfação com a vida & Satisfação diádica \\
\hline Afetos positivos & - & $-0,45(<0,01)$ & $0,19(0,05)$ & $0,09(0,34)$ \\
Afetos negativos & & - & $0,09(0,35)$ & $-0,10(0,33)$ \\
Satisfação com a vida & & & - & $0,20(0,04)$ \\
\hline
\end{tabular}

A avaliação da satisfação com a vida é subjetiva e, como tal, reflete as expressões de cada pessoa quanto aos seus próprios critérios de satisfação com a vida como um todo e em domínios específicos, como saúde, trabalho, condições de moradia, relações sociais e outros. Assim, reflete o bem-estar individual, o modo e os motivos que levam as pessoas a viverem suas experiências de vida de maneira positiva (Resende, Bones, Souza \& Guimarães, 2005). Pode-se compreender esse domínio como um balanço acerca da vida, no qual a pessoa expressa, em forma de julgamento, sua percepção de bem-estar com base em suas experiências positivas e negativas.

A satisfação com a vida não apresenta correlação com o consenso diádico $(\mathrm{r}=0,01 ; \mathrm{p}=0,92)$. Esse resultado não segue a tendência das demais variáveis de BES (afetos positivos e afetos negativos), todas correlacionadas moderadamente com o consenso diádico. Pela análise de regressão múltipla stepwise, a satisfação com a vida mostrou-se significativamente correlacionada com os demais domínios do BES e com o domínio satisfação diádica (Tabela 2). 
Tabela 2 - Resultados do modelo de regressão ajustado utilizando o método de seleção de variáveis stepwise

\begin{tabular}{cccc}
\hline $\begin{array}{c}\text { Variável } \\
\text { resposta }\end{array}$ & $\begin{array}{c}\text { Variáveis } \\
\text { explicativas }\end{array}$ & $\begin{array}{c}\text { Estimativa } \\
\text { do parâmetro }\end{array}$ & p-valor \\
\hline \multirow{3}{*}{ Satisfação com a vida } & Afetos positivos & 0,11 & $<0,01$ \\
& Afetos negativos & 0,06 & 0,01 \\
& Satisfação diádica & 0,24 & 0,03 \\
& Idade & 0,06 & 0,05 \\
\multirow{2}{*}{ Satisfação diádica } & & $-0,05$ & 0,02 \\
& Afetos negativos & 0,15 & 0,05 \\
\hline
\end{tabular}

A variável de conjugalidade denominada satisfação diádica está negativamente correlacionada apenas ao domínio de consenso diádico. Não pode ser associada aos domínios de coesão diádica $(\mathrm{r}=-0,13$; $\mathrm{p}=0,18)$ e expressão do afeto $(\mathrm{r}=-0,14 ; \mathrm{p}=0,16)$, resultado que vai em sentido oposto ao destacado por Spanier (1976), de que esses domínios são correlacionados significativamente. Pela análise de regressão múltipla stepwise, a satisfação diádica não sofre influências de quaisquer das dimensões da conjugalidade mensuradas pelo DAS.

O domínio satisfação, conforme descrito por Spanier (1976) e por Perlin (2006), compreende comportamentos, tais como frequência de conversas ou pensamentos sobre divórcio, separação ou término do relacionamento, frequência com que se pensa que as coisas estão indo bem ou mal no casamento, troca de confidências, de pensamentos sobre possível arrependimento de ter se casado, de irritação com o outro, entre outros.

A partir dessa consideração, pode-se compreender o fato de que a satisfação recebe influência dos afetos positivos, negativos e da própria satisfação com a vida, pela análise de regressão. Se a dimensão é composta pelos pensamentos adaptativos e não-adaptativos relacionados a uma avaliação do que é percebido no casamento e na própria vida, há que se estabelecer a sua relação com os domínios do BES.

A dimensão satisfação com a díade, segundo Perlin (2006), refere-se à percepção direta da satisfação conjugal, como cada cônjuge percebe seu casamento e também em relação à presença de alguns fatores ou comportamentos na vida conjugal satisfatória e nãosatisfatória.

Essa avaliação e percepscão se dá hoje de forma conflituosa, principalmente devido à fase transicional e ao privilégio do individual sobre o relacional e o coletivo. Ainda, é amplificada devido ao contínuo e crescente processo de equidade entre homens e mulheres - ao menos nos discursos. Considerado uma das mais significativas dimensões da vida das pessoas, é normal e saudável que, de tempos em tempos, as pessoas facam análises sobre seus sentimentos e projetos em relação ao casamento. (Perlin, 2006, p. 102)

Desse modo, ainda que tal avaliação contemple uma maior possibilidade de reflexão e de questionamentos acerca do casamento, dos papéis do cônjuge e de como cada um percebe e vivencia o relacionamento conjugal, na contemporaneidade, observa-se que este domínio se correlaciona de modo significativo com todos os domínios da satisfação conjugal, o que pode por causa da consonância entre as escalas e a própria definição dos construtos.

Uma boa avaliação da satisfação no relacionamento diádico deve vir acompanhada de bom nível de afetos positivos, baixo nível de afetos negativos e de um similar grau de satisfação com a vida. Pode-se problematizar tendo em vista que ambas as satisfações (do BES e da conjugalidade) apontam para uma mesma direção, ou seja, avaliar o casamento passaria, inequivocamente, não apenas por uma avaliação da própria vida, mas por uma reflexão acerca do papel do relacionamento afetivo na constituição da pessoa.

Pelos dados obtidos, pode-se dizer que a satisfação diádica está positiva e moderadamente correlacionada com a satisfação com a vida e que pessoas com alto nível de afetos positivos tendem a apresentar, em seus relacionamentos conjugais, maior coesão diádica, maior expressão do afeto pelo cônjuge e maior interação conjugal. Variáveis correlatas, como idade, grau de instrução, renda e tempo de relacionamento não apresentaram associações significativas (Scorsolini-Comin, 2009).

Segundo a literatura, a percepção satisfatória acerca do casamento está diretamente ligada a uma avaliação satisfatória da vida de cada cônjuge e também a um baixo nível de afetos negativos (Lee, Seccombe \& Shehan, 1991; Snyder \& Lopez, 2009). Ou seja, o casamento, avaliado na perspectiva desses domínios, está correlacionado a aspectos individuais trazidos e percebidos por cada cônjuge. Assim, o espaço conjugal atualizaria os aspectos trazidos por cada parceiro, configurando um locus de compartilhamento não apenas de experiências, desejos e sentimentos, mas também de percepções mais ou menos satisfatórias acerca da vida e de suas dimensões, como destacado no estudo de Perlin (2006). Isso reforça a investigação acerca do modo como cada cônjuge se coloca na relação, transferindo suas percepções e posicionamentos não apenas quanto a expectativas acerca do relacionamento conjugal, mas quanto a suas expectativas individuais em relação ao que é ser feliz e como se pode buscar a satisfação, o 
que permite destacar o casamento como uma possibilidade de realização dessa expectativa.

\section{Conclusões}

Considerando os domínios específicos de cada construto, encontrou-se uma correlação moderada e significativa entre a satisfação com a vida (domínio do BES) e a satisfação diádica (domínio da conjugalidade). Esse achado permite afirmar que, na avaliação de bemestar, o relacionamento afetivo ocupa um lugar de destaque, uma vez que é uma fonte significativa de prazer. Retomando uma das problematizações iniciais deste estudo, pode-se destacar que a conjugalidade (notadamente, a satisfação diádica) teria um papel importante na avaliação subjetiva de bem-estar, uma vez que este domínio interfere no modo como cada cônjuge não apenas percebe o seu casamento, mas também como o avalia, tendo referência os demais relacionamentos presentes em seu meio social e cultural, bem como os seus próprios relacionamentos estabelecidos anteriormente. Deve-se destacar, nesse sentido, que os dados refletem um segmento específico da sociedade, constituído de indivíduos casados, de setores urbanos e classe média.

Nos limites do escopo deste estudo, não se pode corroborar a literatura que aponta que pessoas casadas são mais felizes que as demais, mas se pode colocar em relevo, nos participantes casados da amostra, o papel desempenhado pela vivência conjugal na mensuração da própria satisfação com a vida. Futuramente, a avaliação de cada um dos casais acerca desses mesmos domínios poderá revelar se existiria mesmo um BES do casal. Ou seja, dentro de cada casal, quais seriam as correlações entre os domínios de BES? Outra possibilidade que se abre é a de avançar nessa linha de investigação e estudar, do ponto de vista conceitual e estatístico, quais as semelhanças e diferenças entre esse BES do casal e a satisfação conjugal. Pode-se falar em afetos positivos e afetos negativos da relação? Com certeza, ainda há que se galgar espaços para nutrir essas reflexões.

Pela análise dos dados, podemos afirmar que alguns domínios específicos do BES estão ligados a certas dimensões da conjugalidade. Desse modo, os afetos positivos mantêm uma correlação significativa com a coesão e a expressão do afeto, os afetos negativos são relacionados ao consenso e satisfação diádicos e a satisfação com a vida está fortemente correlacionada com a satisfação diádica (hipótese central do estudo).

Assim, o que cada cônjuge transfere para a relação deve ser apreendido como sua contribuição para a construção do espaço conjugal, ou seja, inevitavelmente, seus afetos positivos, afetos negativos e a forma como julga a sua vida fazem parte do seu modo de ser conjugal, isto é, do modo como ele se abre e se desenvolve no seu casamento. Essa ponderação conduz, assim, para a consideração do BES na avaliação do casamento.

Pelos resultados deste estudo pode-se dizer que tais domínios adaptativos e favoráveis ao estabelecimento e manutenção de vínculos estão correlacionados ao que é percebido pelos cônjuges como satisfação. Um exemplo disso é o domínio da satisfação com a vida. Segundo Glatzer (1987) e Costa e Pereira (2007), a satisfação pode ser expressa em diferentes níveis, como a satisfação com a vida, a satisfação com todo um domínio da vida (como acontece com o casamento) e a satisfação com determinados aspectos de um domínio (como ocorre com o parceiro). Um domínio que é menos dependente de situações momentâneas e menos sensível a mudanças súbitas do humor do que a felicidade sofre mais influência de preocupações no espaço de vida mais amplo, como os âmbitos político e social (Seligman, 2004; Snyder \& Lopes, 2009).

O domínio satisfação com a vida, de acordo com os dados do presente trabalho, está positivamente correlacionado com a satisfação diádica, ou seja, a satisfação entre os cônjuges, o que nos permite sugerir que uma vida a dois rica e carregada de elementos e vivências positivas poderia estar diretamente ligada à percepção de satisfação com a vida expressa por cada membro.

De modo similar, uma percepção positiva acerca da vida (uma predominância dos afetos positivos sobre os afetos negativos) pode estar vinculada a altos escores de coesão diádica e de expressão do afeto entre os parceiros. Ou seja, pessoas com altos níveis de afetos positivos tendem a estabelecer relacionamentos nos quais elas podem expressar seus afetos de modo adequado ou nos quais possam interagir a partir da conjugação de aspectos positivos e negativos da própria relação.

Como já destacado por Diener, Suh e Oishi (1998), o nível de satisfação pode ser um modulador das emoções, aumentando ou diminuindo as emoções positivas ou negativas, dependendo do que o indíviduo pensa, o que pode ser estendido à instituição casamento. $\mathrm{O}$ modo como cada cônjuge participa do estar junto em uma relação, compartilhando sentimentos, desapontamentos, diferenças e proximidades, pode ser um fator de satisfação não apenas com o domínio conjugal, como também com a própria vida. Pela correlação encontrada entre os domínios de coesão, consenso, satisfação diádica, expressão do afeto e interação conjugal e os aspectos estruturais do casamento, pode-se sugerir que tais sentimentos e percepções estariam no cerne da estrutura do relacionamento conjugal, uma vez que cada um desses domínios responde pelo que é estar junto, vinculado e experienciando sentimentos de prazer ou desprazer. 


\section{Referências}

Associação Brasileira dos Institutos de Pesquisa de Mercado - Abipeme (1997). Critério de classificação socioeconômica - Brasil (CCSEB).

Albuquerque, A. S. (2004). Bem-estar subjetivo e sua relação com personalidade, coping, suporte social, satisfação conjugal e satisfação no trabalho. Tese de Doutorado. Brasília, DF: Universidade de Brasília - Instituto de Psicologia.

Albuquerque, A. S. \& Tróccoli, B. T. (2004). Desenvolvimento de uma escala de bem-estar subjetivo. Psicologia: Teoria e Pesquisa, 20(2), 153164.

Anguas, A. (1997). El significado del bienestar subjetivo, su valoración en México. Tese de Doutorado. Madrid: Universidad Nacional Autónoma de México.

Argyle, M. (1999). Causes and correlates of happiness. Em D. Kahneman, E. Diener \& N. Schwarz (Orgs.). Well-being: The foundations of Hedonic Psychology (pp. 353-373). Nova Iorque: Russel Sage Foundation.

Carey, M. P., Spector, I. P., Lantinga, L. J. \& Krauss, D. J. (1993). Reliability of the Dyadic Adjustment Scale. Psychological Assessment, 5(22), 238-240.

Costa, L. S. M. \& Pereira, C. A. A. (2007). Bem-estar subjetivo: aspectos conceituais. Arquivos Brasileiros de Psicologia, 59(1), 72-80.

Dias, M. (2000). A construção do casal: um estudo sobre as relaçôes conjugais contemporâneas. Tese de Doutorado. Rio de Janeiro, RJ: Pontifícia Universidade Católica.

Diener, E. \& Lucas, R. E. (2000). Explaining differences in societal levels of happiness: relative standards, need fulfillment, culture and evaluation theory. Journal of Happiness Studies, 1, 41-78.

Diener, E., Suh, E. \& Oishi, S. (1998). Recent findings on subjective well-being. Indian Journal of Clinical Psychology, 2, 25-41.

Emmons, R. A. (1986). Personal strivings: an approach to personality and subjective well-being. Journal of Personality and Social Psychology, 51, 1058-1068.

Fisiloglu, H. \& Demir, A. (2000). Applicability of the Dyadic Adjustment Scale for measurement of marital quality with Turkish couples. European Journal of Psychological Assessment, 16(3), 214-218.

Galinha, I. C. (2008). Bem-estar subjectivo: factores cognitivos, afectivos e contextuais. Coimbra, Portugal: Quarteto.

Glatzer, W. (1987). Subjective well-being: components of well-being. Social Indicators Research, 19, 25-38.
Graham, J. M., Liu, Y. \& Jeziorski, J. L. (2006). The Dyadic Adjustment Scale: a reliability generalization meta-analysis. Journal of Marriage and Family, 68(3), 701-717.

Hernandez, J. A. E. (2008). Avaliação estrutural da escala de ajustamento diádico. Psicologia em Estudo (Maringá), 13(3), 593-601.

Hernandez, J. A. E. \& Hutz, C. S. (2008). Gravidez do primeiro filho: papéis sexuais, ajustamento conjugal e emocional. Psicologia: Teoria e Pesquisa, 24(2), 133-141.

Lee, G. R., Seccombe, K. \& Shehan, C. L. (1991). Marital status and personal happiness: an analysis of trend data. Journal of Marriage and the Family, 53, 839-844.

Magagnin, C., Körbes, J. M., Hernandez, J. A. E., Cafruni, S., Rodrigues, M. T. \& Zarpelon, M. (2003). Da conjugalidade à parentalidade: Gravidez, ajustamento e satisfação conjugal. Aletheia, 17/18, 41-52.

Martinez, M. \& Garcia, M. (1994). La autopercepción de la salud y el bienestar psicológico como indicador de calidad de vida percibida en la vejez. Revista de Psicología de la Salud, 6(1), 55-74.

Montgomery, D., Vining, G. \& Peck, E. A. (2001). Introduction to linear regression analysis. Nova Iorque: John Wiley Profession.

Perlin, G. D. B. (2006). Casamentos contemporâneos: um estudo sobre os impactos da interação família-trabalho na satisfação conjugal. Tese de Doutorado. Brasília, DF: Universidade de Brasilia - Instituto de Psicologia.

Ramos, A. S. \& Patrão, I. (2005). Imagem corporal da mulher com cancro de mama: impacto na qualidade do relacionamento conjugal e na satisfação sexual. Análise Psicológica, 23(3), 295-304.

R Development Core Team (2005). R: a language and environment for statistical computing. R Foundation for Statistical Computing, Vienna, Austria. Obtido do World Wide Web em 10 de outubro de 2008, $<$ http://www.R-project.org $>$.

Resende, M. C., Bones, V. M., Souza, I. S. \& Guimarães, N. K. (2005). Bem-estar subjetivo e rede de relações sociais na vida adulta e velhice. Revista Eletrônica da Sociedade de Psicologia do Triângulo Mineiro, 9(1), 9-16.

Scorsolini-Comin, F. (2009). Casar, verbo (in)transitivo: conjugalidade, bem-estar subjetivo e satisfação conjugal na perspectiva da Psicologia Positiva. Dissertação de Mestrado. Ribeirão Preto, SP: Universidade de São Paulo - Faculdade de Filosofia, Ciências e Letras de Ribeirão Preto. 
Seligman, M. E. (2004). Felicidade autêntica: usando a nova psicologia positiva para a realizaşão permanente. Rio de Janeiro: Objetiva.

Shin, D. C. \& Johnson, D. M. (1978). Avowed happiness as an overall assessment of the quality of life. Social Indicators Research, 5, 475-492.

Snyder, C. R. \& Lopez, S. J. (2009). Psicologia positiva: uma abordagem cientifica e prática das qualidades bumanas. (R. C. Costa, trad.). São Paulo: Artmed.
Spanier, G. B. (1976). Measuring dyadic adjustment: new scales for assessing quality of marriage and similar dyads. Journal of Marriage and the Family, 38(1), 15-28.

Zou, K. H., Tuncali, K. \& Silverman, S. G. (2003). Correlation and simple linear regression. Radiology, 227, 617-628.

Recebido em novembro de 2009

Reformulado em janeiro de 2010

Aprovado em maio de 2010

Sobre os autores:

Fabio Scorsolini-Comin: é psicólogo, mestre e doutorando em Psicologia pela Faculdade de Filosofia, Ciências e Letras de Ribeirão Preto, Universidade de São Paulo (FFCLRP -USP); especialista em Gestão da Educação: Administração Escolar e em Gestão da Educação: Supervisão Educacional e bolsista de Doutorado da CAPES.

Manoel Antonio dos Santos: é psicólogo, mestre e doutor em Psicologia Clínica pela Universidade de São Paulo (USP); professor doutor do Departamento de Psicologia e Educação da Faculdade de Filosofia, Ciências e Letras de Ribeirão Preto, Universidade de São Paulo (FFCLRP USP) e bolsista de Produtividade em Pesquisa do CNPq.

Agradecimentos:

Trabalho derivado da dissertação de mestrado intitulada "Casar, verbo (in)transitivo: bem-estar subjetivo, conjugalidade e satisfação conjugal na perspectiva da Psicologia Positiva", do primeiro autor, sob orientação do segundo autor, defendida em 2009 junto ao Programa de Pós-Graduaçăo em Psicologia da Faculdade de Filosofia, Ciências e Letras de Ribeirão Preto da Universidade de São Paulo. Os autores agradecem à Fundação de Amparo à Pesquisa do Estado de São Paulo (FAPESP) por ter subvencionado o projeto que originou o presente estudo, com bolsa de mestrado (Processo $n^{\circ}$ 2007/52584-5), e ao Conselho Nacional de Desenvolvimento Científico e Tecnológico (CNPq) pela bolsa de Produtividade em Pesquisa, nível 1D, concedida ao segundo autor. 